\title{
Performance Dashboards als Steuerungsinstrument
}

Das Energieunternehmen Vattenfall hat im Bereich Performance Management Dashboards eingeführt, um seine Unternehmensstrategie besser umsetzen zu können. Diese Dashboards ermöglichen Management und Controlling eine agile Leistungsmessung und -steuerung. Damit dies gelingt, mussten bei der Konzeption und Implementierung grundlegende Schritte beachtet werden.

Robert Zurawski, Axel Neumann-Giesen, Andreas Rauh 
Schnelles und gleichzeitig starkes Wachstum, steigender Wettbewerbsdruck und volatile Marktbedingungen erfordern eine agile Informationsbasis, die dem Management einen holistischen Blick auf die Performance eines Unternehmens gewährt. Das europäische Energieunternehmen Vattenfall mit Sitz in Schweden steht genau dieser Herausforderung gegenüber. Vattenfall agiert in den Bereichen Erzeugung, Distribution und Vertrieb von Strom, Wärme und Gas. Sein erklärtes strategisches Ziel ist es, innerhalb einer Generation eine fossil-freie Energieversorgung zu ermöglichen. Um dies zu erreichen, liegt der aktuelle Fokus des Managements auf dem Ausbau des Sektors der erneuerbaren Energien und dem sukzessiven Rückbau des fossilen Sektors. Besonders der Geschäftsbereich (Business Area - BA) Wind, der für die Entwicklung, den Bau und Betrieb von Windkraftanlangen an Land (Onshore) und auf hoher See (Offshore), Solaranlagen und Batteriespeichern zuständig ist, soll maßgeblich zum Ausbau erneuerbarer Energien beitragen.

Um diesen Wandel steuern zu können, rückt das Thema Performance Management (PM) unternehmensweit und im Speziellen auch bei der BA Wind immer stärker in den Fokus. Mit der Unterstützung durch CTcon Management Consultants hat Vattenfall 2019 Management Dashboards im Rahmen von Power BI, dem Business Intelligence Tool von Microsoft, entwickelt und implementiert. Diese Dashboards haben zum Ziel, das laufende Geschäft und neue Investitionsprojekte möglichst effizient zu steuern. Aufbauend auf einer einheitlichen Datenbasis, fördern sie faktenbasierte und maßnahmenorientierte Diskussionen im Top-Management. Komprimiert auf einer Seite gewähren digitale Dashboards einen strukturierten Überblick steuerungsrelevanter Informationen - und das zu jeder Zeit und an jedem Ort.

Damit dies gelingen konnte, wurden bei der Konzeption und Implementierung der Dashboards sechs Schritte berücksichtigt.

\section{Schritt 1: Steuerungsrelevante KPIs und Treiber identifizieren}

Die Definition von Top-KPIs und deren Treibern ist ein erfolgsentscheidender Schritt bei der Konzeption von Management Dashboards. Da die heutigen operativen IT-Systeme wie beispielsweise ERP große Mengen an Daten liefern, ist eine Reduktion auf wenige, steuerungsrelevante KPIs notwendig, um einer „Informationsflut" vorzubeugen. Eine optimale Anzahl an KPIs je Dashboard liegt dabei erfahrungsgemäß zwischen acht und zwölf. Sind diese identifiziert, werden die KPIs mit den wesentlichen Treibern hinterlegt. Diese Haupttreiber bilden die Grundlage für fokussierte Analysen von Performance-Problemen und helfen dabei, Ursache-Wirkungs-Zusammenhänge transparent zu machen. Mit den relevanten KPIs und Treibern erhält das Management einen strukturierten Überblick über die für die Steuerung wesentlichen Informationen. Es kann besser nachvollziehen, woraus Abweichungen der KPIs resultieren, über konkrete Möglichkeiten der Einflussnahme diskutieren und Maßnahmen ableiten.

Um eine fokussierte Anzahl von steuerungsrelevanten KPIs für alle Bereiche der BA Wind bei Vattenfall zu identifizieren, wurden Manager der ersten bei-

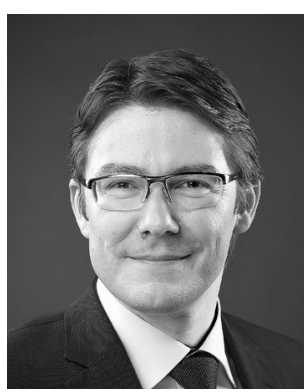

\section{Robert Zurawski}

ist Vice President Business Control, Business Area Wind bei Vattenfall, Berlin.

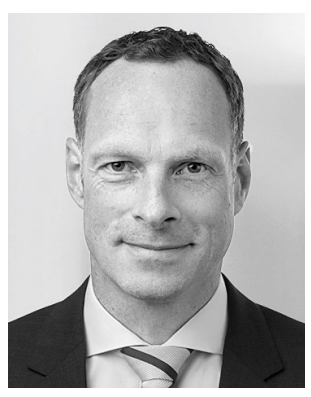

\section{Axel Neumann-Giesen}

ist Partner bei CTcon Management Consultants, Bonn.

E-Mail:a.neumann-giesen@ctcon.de

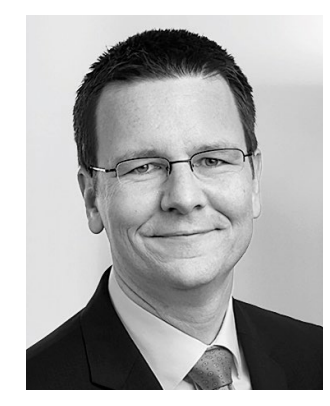

Andreas Rauh

ist Principal bei CTcon Management Consultants, München.

E-Mail:a.rauh@ctcon.de 
den Führungsebenen sowie die Business Controller in Einzel-Interviews nach ihren Anforderungen befragt. Die Auswahl erfolgte anhand von zwei Kriterien:

1. KPI-Sets zeigen nur dann ein holistisches und aussagekräftiges Bild, wenn alle relevanten Steuerungsbereiche abgedeckt sind. Dazu gehört die Abbildung von finanziellen Kennzahlen zur Messung der Profitabilität sowie Kennzahlen zur Messung von Produktivität und Qualität. Zudem müssen alle Unternehmensbereiche abgebildet werden.

2. Einzelne KPIs haben spezifische Gütekriterien zu erfüllen, um als sinnvolle Messgröße zu gelten. Sie müssen steuerungsrelevant und beeinflussbar sein, einen materiellen Tatbestand abbilden und einer klaren persönlichen Verantwortlichkeit zuordenbar sein.

Nachdem KPI-Sets auf diese Weise systematisch für die ersten beiden Management-Ebenen erarbeitet worden sind, wurden gemeinsam mit dem Geschäftsbereich und dem Controlling die wichtigsten Werttreiber der KPIs ergänzt. Damit stand die inhaltliche Basis für die Erstellung der Dashboards. Das Beispiel der Kennzahl „Revenue Based Availability (RBA)“, einer operativen Steuerungsgröße der BA Wind, veranschaulicht exemplarisch den Aufbau eines Werttreiberbaumes (vergleiche Abbildung 1).

\section{Schritt 2: Grafische Standards festlegen}

Sind die relevanten KPIs und Treiber je Bereich festgelegt, folgen Überlegungen zur Gestaltung der Dashboards. Ziel sollte es sein, eine einheitliche und intuitiv zu bedienende Nutzeroberfläche zu schaffen. Die Grafiken müssen leicht lesbar und verständlich sein, damit relevante Informationen für

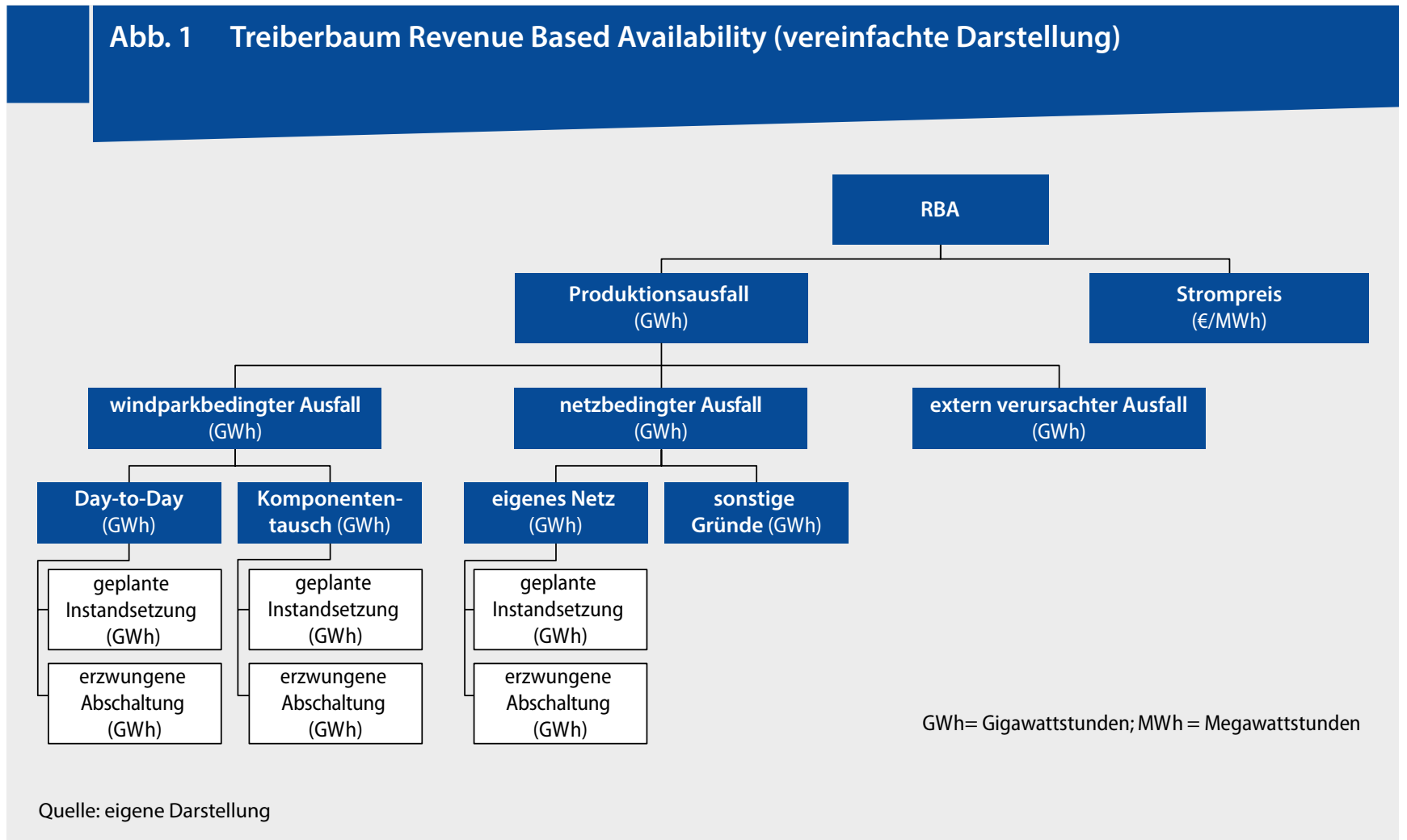


den Dashboard-Nutzer sofort sichtbar sind. Als Grundvoraussetzung sollten einheitliche Farbcodes und Grafikelemente definiert und eine standardisierte Gestaltung der Navigationsfelder festgelegt werden.

Bei der BA Wind einigte man sich für die Management Dashboards auf Tachometer als Grafikelement und eine ergänzende Ampel-Farbskala, um PlanIst-Abweichungen zu visualisieren (vergleiche Abbildung 2). Planabweichungen werden besonders hervorgehoben, indem das absolute Delta unterhalb des jeweiligen Graphen abgebildet und je nach Richtung und Höhe in Ampellogik gefärbt wird. Insgesamt sind die Dashboards in mehrere Felder unterteilt: Das rechte Drittel der Dashboards ist variabel gestaltet und über eine Schaltfläche steuerbar. Es zeigt entweder ergänzende KPIs zu den Top-Steuerungsgrößen oder das sogenannte „Storyboard“, das zusätzliche erläuternde Kommentare für das Management enthält. In einem Kommentar kann beispielsweise die operative Ursache (Kabelbruch), die zu einer Abweichung bei RBA führt, geschildert und die eingeleiteten Maßnahmen beschrieben werden.

Auf separaten „Deep-Dive-Seiten“, welche per Klick auf den jeweiligen KPI zu erreichen sind, sind Details je KPI, wie beispielsweise Verläufe, thematische Aufbrüche oder Werttreiber, abrufbar. Dabei werden Liniendiagramme für Verlaufsdarstellungen, Wasserfall-Charts für Delta-Betrachtungen und Datentabellen für Detailinformationen verwendet.

\section{Die Definition steuerungs- relevanter KPIs ist eine Grundvoraussetzung für den Aufbau von Dashboards.}

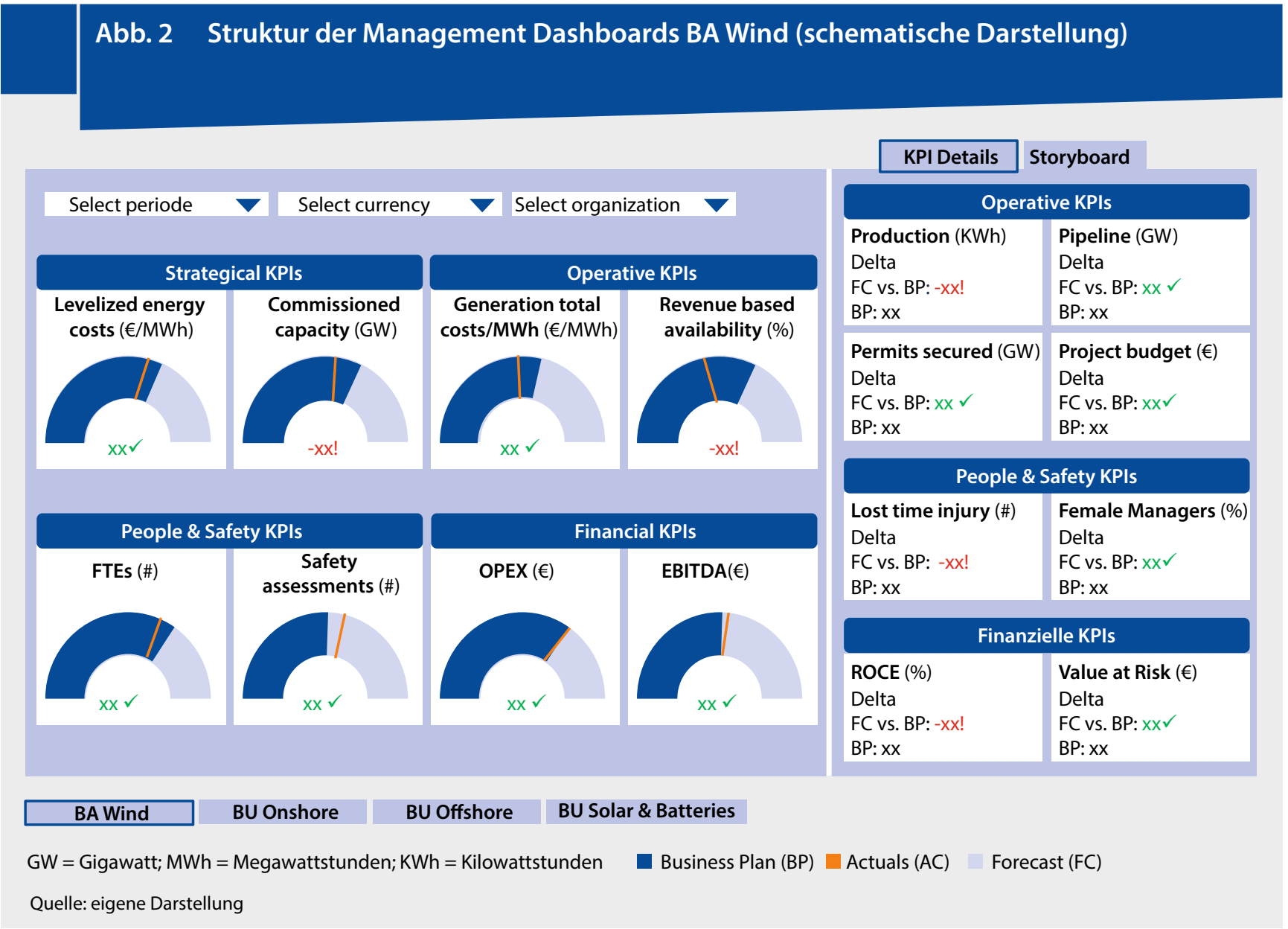




\section{Die nachhaltige Gestaltung einer konsistenten Datenbasis gelingt nur mit ausreichenden IT-Ressourcen.}

\section{Zusammenfassung}

- Ein wettbewerbsintensives Geschäftsumfeld macht es unumgänglich, Entscheidungen auf einer soliden Datengrundlage, treiberbasiert und möglichst in Echtzeit treffen zu können.

- Um dies zu ermöglichen, hat das Energieunternehmen Vattenfall mithilfe der Management-Beratung CTcon das Reporting des Geschäftsbereichs Wind digitalisiert und eine Dashboard-Lösung konzipiert und implementiert.

- Die Dashboards zeigen übersichtlich und fokussiert die wesentlichen steuerungsrelevanten KPIs auf und ermöglichen einen Drill-down auf die dahinterliegenden Treiber.
Durch die standardisierte Gestaltung der Nutzeroberfläche wird sichergestellt, dass sich Manager und Controller auf allen Dashboards und DeepDive-Seiten schnell zurechtfinden. Steuerungsrelevante Informationen können sofort identifiziert werden; eine Erläuterung der Abbildungen ist nicht notwendig. Über Reiter an der Unterkante der Dashboards sind Übergänge von der BA Wind auf die nachgeordneten Business Units (BU) in gleichem Look and Feel (Aussehen und Handhabung) angelegt.

\section{Schritt 3: Aufbau und Zusammenspiel der Dashboards konzipieren}

Dashboards bieten den Nutzern die Möglichkeit, Ursachen und Ursprüngen von Performance-Problemen auf den Grund zu gehen. Dies stellt einen großen Vorteil gegenüber starren Offline-Berichten dar. Mithilfe von Drilldown und Drillup-up können KPIs hierarchisch verfeinert analysiert werden. Es ist beispielsweise ein Aufriss nach Ländern oder Regionen möglich, wobei auch wieder auf eine aggregierte Ebene zurückgesprungen werden kann. Mithilfe von Deep Dives können Treiber der KPIs betrachtet werden. Die Dashboard-Landschaft sollte so flexibel aufgebaut sein, dass jederzeit zusätzliche Informationen und Auswertungen hinzugefügt werden können. Eine standardisierte Navigationsführung schließlich trägt zur leichteren Handhabung der Dashboards und einem raschen Zurechtfinden der Nutzer bei, da Self-Service-Analysen stets auf dieselbe Art und Weise erfolgen.

Um diese Vorteile voll ausschöpfen zu können, wurde in der BA Wind ein detailliertes Konzept zur Gestaltung der Dashboard-Landschaft erarbeitet. Als Startpunkt wurde das Bereichs-Dashboard des jeweiligen Business Unit Managers gewählt, das einen Überblick über die Gesamt-Performance bietet. Durch einen Drop-down-Filter können die jeweiligen Felder der organisatorischen Einheiten ausgewählt und Planabweichungen lokalisiert werden. Umgekehrt ist ein Drill-up möglich, welcher Auskunft über den Beitrag der eigenen Performance auf die nächsthöhere Ebene gibt. Für eine Ursachenanalyse gelangen Manager jeweils durch einen Klick auf den KPI auf eine separate Deep-Dive-Seite mit weiteren Details.

Durch eine organisationale Drill-down-Navigation ist eine konsequente Kaskadierung der KPIs entlang der Organisation möglich. Akute Performance-Probleme sind sofort erkennbar; integrierte Deep-Dive-Möglichkeiten schaffen Transparenz über Ursachen und bieten eine optimale Informationsbasis zur Ableitung von Maßnahmen. Der Zugriff auf die Dashboards überall und jederzeit bringt Flexibilität und macht das manuelle Versenden von Reports obsolet.

Um auf veränderte Geschäftsverhältnisse zu reagieren oder neue Themen nachhaltig nachzuvollziehen, ergänzt die BA Wind klassische Management Dashboards mit Sonderauswertungen. So wurde beispielsweise ein sogenannter „Covid-19 Action Tracker“ integriert, um definierte Maßnahmen nachverfolgen zu können. Für große strategierelevante Projekte werden zudem spezifische Steuerungs-Cockpits in die Dashboard-Landschaft aufgenommen. 


\section{Schritt 4: Konsistente Datenbasis sicherstellen}

Damit das Management wichtige erfolgsrelevante Zusammenhänge leichter erkennen und seinen Geschäftsbereich zielgerichtet, effizient und effektiv steuern kann, müssen bestimmte technische Voraussetzungen erfüllt werden. Die Digitalisierung des Reportings beschränkt sich demnach nicht auf die Konzeption von Dashboards und anschließender Implementierung. Ein Großteil der Arbeit findet in der Aufbereitung der Daten für eine konsistente Datenbasis und dem Aufbau einer nachhaltigen Backend-Lösung statt. Die automatisierte Anbindung von Datensystemen an ein digitales Frontend kann nur dann für höchstmögliche Aktualität der Daten sorgen und den manuellen Aufwand für die Berichtserstellung eliminieren, wenn Daten aus unterschiedlichen Systemen in einem Data Warehouse miteinander verknüpft werden. Dadurch werden Abhängigkeiten transparent, die bisher nicht oder nur unter hoher Anstrengung ersichtlich waren. Eine ausreichende Einplanung von IT-Ressourcen ist deshalb bei der Projektplanung unbedingt vorzunehmen.

In der BA Wind hat man sich dazu entschieden, den Aufbau einer stringenten Datenbasis in einem eigenständigen IT-Projekt in Abstimmung mit dem IT-Bereich des Vattenfall-Konzerns voranzutreiben. Dies stellt sicher, dass an einer nachhaltigen Backend-Lösung gearbeitet wird und keine Insellösung entsteht. Vor der Dashboard-Lösung hat die BA Wind Excel-Auswertungen genutzt. Aufgrund deren Instabilität wurde entschieden, diese zum Großteil in eine Datenbanklösung zu überführen. Das hat auch den Vorteil, dass Daten nicht länger rückwirkend verändert werden können; alle erfassten Informationen erhalten einen Zeitstempel. Vor der Einführung der DashboardLösung lagen Daten aus existierenden Systemen wie SAP häufig im „Rohformat" vor und mussten entsprechend manuell aufbereitet werden. Beispielsweise wurde die Profit-and-Loss-Kalkulationslogik hinterlegt oder konsolidierungsrelevante interne Verrechnungen wurden manuell eliminiert. Jetzt werden dem Prinzip der „Single Source of Truth“ folgend KPI-Kalkulationen oder Konsolidierungen möglichst direkt in den Ursprungsdatenquellen durchgeführt. Dies stellt sicher, dass KPIs unternehmensweit einheitlich vorliegen. Zudem können mehrere Nutzer gleichzeitig auf die Basisdaten zugreifen.

\section{Schritt 5: Nutzerkonzept erarbeiten und implementieren}

Eine Dashboard-Lösung wird zum Erfolg, wenn sie zum einen von den Nutzern, für die sie gedacht ist, akzeptiert wird und zum anderen der Zugriff auf das Dashboard eindeutig geregelt ist. Es muss klar definiert sein, wer auf welche steuerungsrelevanten Informationen Zugriff hat. Ein im Frontend hinterlegtes Nutzerkonzept gewährleistet dies bei Vattenfall. Unterschieden wird zwischen einem allgemeinen Dashboard-Zugriff, der rollenabhängig ist (Role-based Security), und der Berechtigung, auf einzelne (vertrauliche) Informationen zuzugreifen (Row-based Security). So kann beispielsweise einem Manager der Zugriff auf das Dashboard eines anderen Bereiches gewährt werden, ohne dass er gleichzeitig Zugriff auf sensible personenbezogene Informationen erhält.
Eine kontinuierliche ChangeBegleitung aller Stakeholder fördert den Erfolg einer Dashboard-Implementierung. 


\section{Schritt 6: Change Management integrieren}

Dashboards gut zu konzipieren und zu implementieren garantiert dennoch nicht, dass sie von den Nutzern in der Praxis auch akzeptiert, angewendet und richtig eingesetzt werden. Damit die veränderte Reporting-Landschaft Manager optimal bei der operativen und strategischen Steuerung unterstützen kann, ist deshalb eine kontinuierliche und intensive Change-Begleitung von Dashboard-Projekten notwendig.

Aus diesem Grund hat die BA Wind ein intensives und agiles ErwartungsManagement je Stakeholder-Gruppe betrieben (vergleiche Abbildung 3). Neben kontinuierlichen Projekt-Updates wurden früh Showcases/Anwendungsbeispiele entwickelt und direkt in Performance Meetings verprobt. Erste Erfahrungen mit den Dashboards wurden gesammelt, Feedback direkt aufgenommen und bei der weiteren Implementierung berücksichtigt. Wesentlich zur Akzeptanz hat beigetragen, dass die BU Manager „ihre“ Dashboards selbst in den Performance Meetings mit der BA vorstellten und die Dashboard-Logik in den Abteilungen der BU aktiv vorantrieben. Dadurch wurden Stakeholder direkt einbezogen und sukzessive auf anstehende Veränderungen vorbereitet.

Um zukünftige Nutzer auf die neue Arbeitsweise vorzubereiten, wurden anwenderspezifische Trainings angeboten. Controller erhielten eine Einführung in die technischen Funktionalitäten, Manager in den Umgang mit dem Tool, zum Beispiel unterschiedliche Analysemöglichkeiten, sowie zu neuen

\section{Abb. 3 Change-Formate je Stakeholder-Gruppe (schematische Darstellung)}

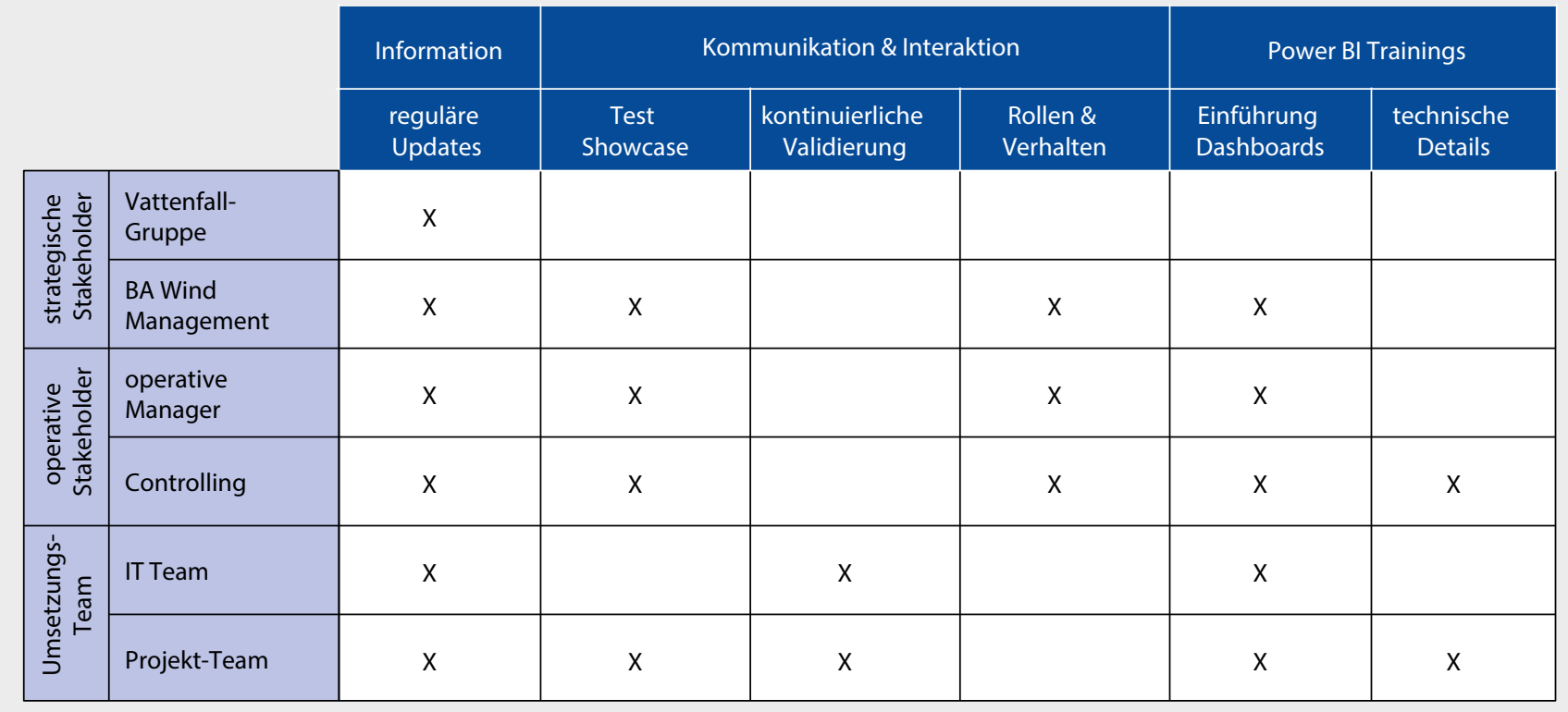

Quelle: eigene Darstellung 
Verhaltensanforderungen. Zudem wurden Regeln zu maximalen DetailLevels von Deep Dives und konkrete Zeitvorgaben für die Diskussion je Thema festgelegt. Dies sollte verhindern, dass Ad-hoc-Analysen im Meeting zu überlanger Befassung mit Einzelsachverhalten führten.

Eine enorme kulturelle Herausforderung stellt die hohe Transparenz der Daten dar. Gemäß der „Single Source of Truth“ ist ein vorhergehendes Bereinigen und „Beschönigen“ von Daten oder ein Filtern von Informationen nicht länger möglich. Die einzelnen Bereiche und Nutzer müssen dazu bereit sein, ihre Daten im Unternehmen zu teilen. Um dies sicherzustellen, muss eine Kultur der Offenheit, der Konstruktivität, des Vertrauens und gegenseitigen Förderns etabliert werden. Nur ein Leben dieser Werte in der täglichen Arbeit kann eine solche kulturelle Veränderung herbeiführen. Das Top-Management nimmt dabei eine Vorbildfunktion ein und ist mitverantwortlich, diesen Wandel voranzutreiben. Nur so kann verhindert werden, dass trotz ausgereiften Konzepts und konsistenter Datenbasis die Einführung von Dashboards aufgrund kultureller Barrieren scheitert. Regelmäßige Information, aktiver Einbezug, ausreichende Trainings-Angebote sowie Vorleben des Wandels durch das Top-Management müssen auch über die Projektlaufzeit hinaus aktiv betrieben werden. Entsprechende Ressourcen und Formate sind von Beginn an einzuplanen.

\section{Fazit}

Das Dashboard-Projekt von Vattenfall BA Wind und CTcon hat gezeigt, dass Dashboards Enabler für ein erfolgreiches Performance Management darstellen. Die Erfahrungen bei der Konzeption und Implementierung haben deutlich gemacht, dass die Digitalisierung des Reportings verschiedenen Erfolgsfaktoren unterliegt. Maßgeblich für ein erfolgreiches Projekt sind ein klares und nutzerbezogenes Konzept zu KPIs, Dashboards und Deep Dives, ausreichend IT-Ressourcen sowie eine kontinuierliche Change-Begleitung unter Einbeziehung des Top-Managements. Sind diese Aspekte berücksichtigt, können digitale Dashboards ihr volles Potenzial unter Beweis stellen und das Management optimal mit steuerungsrelevanten Informationen ausstatten - jederzeit, an jedem Ort und flexibel auswertbar.

\section{SpringerProfessional}




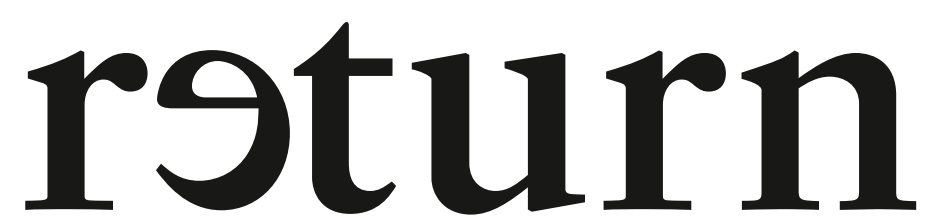

Magazin für Transformation und Turnaround

\section{Schutz gegen den freien Fall}

Beim Bewältigen von Unternehmenskrisen oder noch besser beim Verhindern eines möglichen freien Falls halten Vorstände und Geschäftsführer besonders viel Verantwortung und Risiko in ihren Händen. Clevere Unternehmer und Manager sorgen vor: Gegen Krisen sind sie gewappnet mit klugen Lösungen für erfolgreiches Handeln für Transformation und Turnaround.

return - das Magazin für Manager, die Krisen besser meistern.

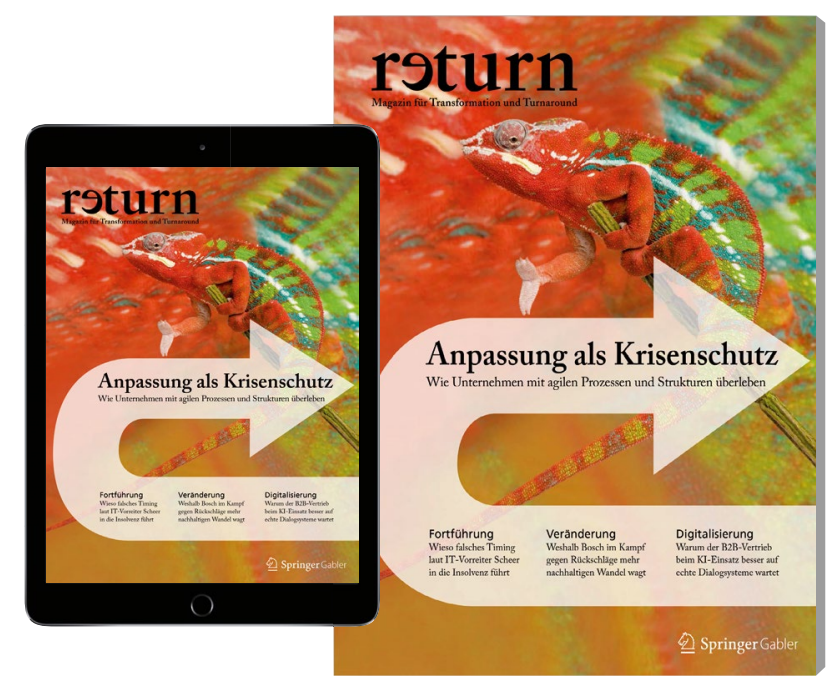

Springer Gabler 